\title{
Modulation of Embryonic Chick Motoneuron Glutamate Sensitivity by Interneurons and Agonists
}

\author{
Richard J. O'Brien' ${ }^{1}$ and Gerald D. Fischbach \\ Department of Anatomy and Neurobiology, Washington University School of Medicine, St. Louis, Missouri 63110
}

Embryonic chick motoneurons grown in culture together with other spinal cord cells are more sensitive to L-glutamate than are sorted motoneurons grown in isolation. After $6 \mathrm{~d}$ in vitro, the difference in peak sensitivity reached 6-fold. Comparable increases in aspartate and kainate currents were observed, indicating that both $G_{1}$ and $G_{2}$ amino acid receptors were affected. Elimination of proliferating non-neuronal cells from mixed spinal cord cell cultures by addition of cytosine arabinoside (ara C) did not prevent the increase in motoneuron chemosensitivity, so the induction is probably due to the presence of interneurons. In contrast to their effect on glutamate response, interneurons did not affect the sensitivity of motoneurons to the inhibitory neurotransmitters GABA and glycinc.

Glutamate receptors expressed by sorted and unsorted motoneurons are identical in terms of their $\mathbf{E D}_{50}$, reversal potential, mean channel open time, and conductance, implying that the increased sensitivity of motoneurons in mixed cultures is due to an increase in the number of open channels.

In addition to an increase in the number of channels, the distribution of glutamate sensitivity over the surface of individual motoneurons was altered in interneuron-containing cultures. The sensitivity of isolated motoneurons was greatest at the soma and decreased with distance along major processes, but the sites of highest sensitivity on motoneurons in mixed cultures occurred along their processes. Sharp peaks identified by focal iontophoresis of glutamate were separated by areas of lower sensitivity.

The inductive effect of interneurons cannot be due to glutamate, the most likely excitatory interneuron-motoneuron transmitter in $6 \mathrm{~d}$ chick cultures. On the contrary, glutamate sensitivity was inversely related to the effective concentration of glutamate in the medium. Chronic exposure to selective agonists and antagonists showed that $G_{1}$ and $G_{2}$ receptors could be regulated independently.

Embryonic motoneurons promote the accumulation of $\mathrm{ACh}$ receptors in the postsynaptic membrane of target myotubes (see reviews by Cohen, 1980; Fambrough, 1979; Fischbach et al., 1979; Steinbach and Bloch, 1986). This phenomenon has been studied extensively because the transmitter, $\mathrm{ACh}$, has been identified, and we know a great deal about the function and structure of ACh receptors. Also, it is possible to isolate embryonic my-

Received Jan. 14, 1986; revised Apr. 25, 1986; accepted May 5, 1986.

We are grateful to Dr. Robert Miller and Ms. Judith Dodge for performing the glutamate assays, to Drs. David Gottlieb and Steven Rothman for suggesting the use of GPT, and to Dr. Charles Zorumski for helpful discussions. This work was supported by USPHS Grant NS 18458 (G.D.F.) and by Harvard Medical School Medical Scientist Training Program I 32 GM07753 (R.O.).

Correspondence should be addressed to Gerald D. Fischbach, Department of Anatomy and Neurobiology, Washington University School of Medicine, 660 South Euclid Ave., St. Louis, MO 63110.

Present address: Department of Medicine, Massachusetts General Hospital, Fruit Street, Boston, MA 02114.

Copyright (c) 1986 Society for Neuroscience $0270-6474 / 86 / 113290-07 \$ 02.00 / 0$ otubes in vitro and analyze the distribution of $\mathrm{ACh}$ receptors both before and after they are innervated. In comparison, very little is known about the influence of presynaptic neurons on the accumulation of chemoreceptors in target neurons within the CNS. In most cases, the transmitter is not known with certainty, and the postsynaptic receptors have not been characterized in detail. Moreover, it is difficult to study a single embryonic neuron in isolation from others that provide synaptic input.

We have shown that embryonic chick motoneurons, labeled in vivo with fluorescein-wheat germ agglutinin (Fl-WGA) conjugates, can be sorted away from other spinal cord cells and grown for at least a week in culture (O'Brien and Fischbach, 1986a). We have also shown that when motoneurons are grown together with interneurons they receive excitatory and inhibitory synaptic input. The excitatory transmitter is probably L-glutamate, and during the first week in culture, this amino acid activates 2 types of postsynaptic receptors termed $G_{1}$ and $G_{2}$ (O'Brien and Fischbach, 1986b, c). We report here that interneurons increase the number of motoneuron $G_{1}$ and $G_{2}$ receptors and alter their distribution. In contrast, the number of receptors for the inhibitory amino acid GABA is unaffected by the presence of interneurons.

\section{Materials and Methods}

\section{Cell cultures}

Techniques for labeling embryonic day (E) 5 chick motoneurons by retrograde transport of fluorescent tracers, for identifying them in heterogeneous spinal cord cell cultures, and for isolating them from other spinal cord cells with a Becton-Dickinson fluorescence-activated cell sorter (FACS IV) have been described (O'Brien and Fischbach, 1986a). The spinal cords were dissociated on F6 a day after labeling to optimize the survival of sorted and unsorted motoneurons; they were plated in dishes containing myotubes and fed with Eagle's Minimum Essential Medium supplemented with $2 \%(\mathrm{vol} / \mathrm{vol})$ embryo extract as wcll as horse serum, glutamine, and antibiotics (O'Brien and Fischbach, 1986a). The medium was changed on day 4 and again on day 6 . In some experiments, proliferating non-neuronal cells were eliminated with $10^{-5}$ M cytosine arabinoside (ara $\mathrm{C}$ ). The antimitotic was added to the medium on the 1st day after plating and removed on the 3rd.

Spinal cord cell conditioned medium was prepared by centrifuging medium removed from $4 \mathrm{~d}$ spinal cord cultures at $800 \times g$ for $10 \mathrm{~min}$. Conditioned medium was added at full strength or diluted 1:1 with fresh medium to cultures of sorted motoneurons every $24 \mathrm{hr}$.

In some experiments, amino acid receptor antagonists and agonists were added to the medium. Gamma-D-glutamylglycine $(\gamma \mathrm{GG} ; \mathrm{Cam}$ bridge Research Biochemicals), 2-amino-5-phosphonovaleric acid (2APV; Cambridge Research Biochemicals), or kainic acid was added in fresh medium every $24 \mathrm{hr}$ between days 1 and 4 in vitro. These compounds apparently were not degraded significantly: medium removed after $24 \mathrm{hr}$ was as potent in inhibiting or exciting (in the case of kainic acid) motoneurons as fresh medium containing the same agents. In other experiments, glutamate levels were reduced by omitting glutamine from the medium and adding the enzyme glutamate pyruvate transaminase (EC 2.6.1.2) at $10 \mathrm{units} / \mathrm{ml}$ along with $10 \mathrm{~mm}$ pyruvate each day. GPT, 

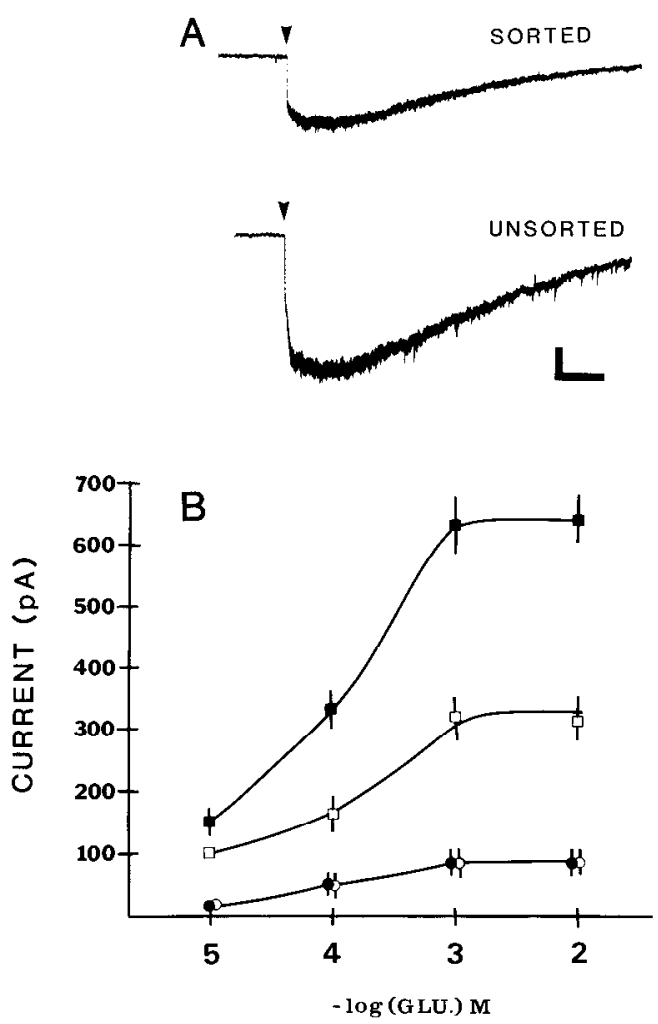

Figure 1. Inward currents evoked by glutamate in sorted and unsorted motoneurons voltage clamped at $-50 \mathrm{mV}$. $A$, Typical responses in 2 motoneurons tested $4 \mathrm{~d}$ after plating. Glutamate was applied at $10^{-3} \mathbf{M}$ by pressure ejection $(0.5 \mathrm{sec}$ pulse) at the arrowheads. Upper trace, sorted motoneuron; lower trace, motoneuron grown with other spinal cord cells. Calibration bars: $25 \mathrm{pA}, 1 \mathrm{sec} . B$, Dose-response curve determined on day 4 (open symbols) and day 6 (closed symbols). Squares represent motoneurons in heterogeneous spinal cord cultures, while circles represent sorted motoneurons. Each point represents the mean \pm SEM of at least 12 cells from 3 platings or sorts. Each motoneuron was tested at a single concentration of glutamate.

prepared from pig heart, was obtained from Sigma Chemical Co. The glutamate concentration in culture medium under different conditions was kindly determined by Dr. R. Miller using $O$-phthaldialdehyde precolumn derivatization and reverse-phase high-performance liquid chromatography techniques as described by Jones and Gilligan (1983).

\section{Electrophysiology}

Patch-clamp microelectrode techniques were identical to those described in O'Brien and Fischbach (1986b). Glutamate was applied by pressure ejection or by iontophoresis. Iontophoretic electrodes measured approximately $100 \mathrm{M} \Omega$ when filled with $0.5 \mathrm{M}$ glutamate at $\mathrm{pH}$ 8. Synaptic activity was suppressed by reducing extracellular $\mathrm{Ca}^{2+}$ to $0.1 \mathrm{~mm}$ and increasing $\mathrm{Mg}^{2+}$ to $5.0 \mathrm{~mm}$. Brief (3-5 msec) negative pulses of current (100-200 nA) were used to map the chemosensitivity of motoneurons. Although a positive backing current of about $2 \mathrm{nA}$ was employed in some instances, it was usually not needed. A camera lucida drawing was made of each motoneuron, and then the maximum inward current at several sites on the soma and at sites separated by 10-25 $\mu \mathrm{m}$ along one or more of the cell's major processes was determined with a constant ejection pulse. Because of the relatively low glutamate sensitivity of sorted motoneurons, we selected the most responsive (sorted) cells for iontophoretic mapping.

\section{Results}

Regulation of motoneuron glutamate sensitivity by interneurons Sorted motoneurons were less sensitive to L-glutamate than were unsorted motoneurons grown with other spinal cord cells. Figure $1 A$ shows typical inward currents induced by $10^{-3} \mathrm{M}$ glutamate
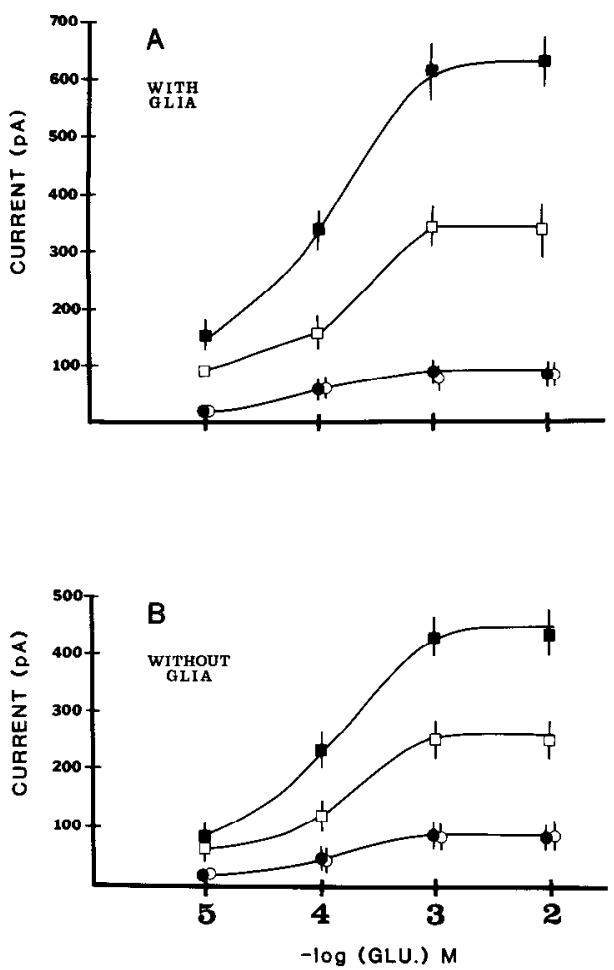

Figure 2. Non-neuronal cells are not responsible for the increase in motoneuron glutamate chemosensitivity. The sensitivity of sorted (circles) and unsorted (squares) motoneurons to L-glutamate was assayed on days 4 (open symbols) and 6 (closed symbols) in vitro. A, Sorted motoneurons were grown on a confluent bed of flat non-neuronal cells prepared by passaging cells dissociated from E15 spinal cords. $B$, Proliferating non-neuronal cells were eliminated from unsorted spinal cord cell cultures by exposure to $10^{-5} \mathrm{M}$ ara $\mathrm{C}$ as described in Materials and Methods. Each point represents the mean \pm SEM of 10 neurons from 3 platings or sortings.

in 1 sorted and 1 unsorted motoneuron from sister cultures plated at the same time. The dose-response curves (Fig. 1B) show that the difference in maximal response was about 3 -fold after $4 \mathrm{~d}$ in culture (open symbols); by day 6 , it had increased 6 -fold (closed symbols). Although the curves are based on only 4 points, they indicate that the increased maximal response was not associated with a shift in the $E D_{50}$.

Both $G_{1}$ and $G_{2}$ receptors contributed to the increase in total glutamate current (Table 1). By day 6 in vitro, the peak response of motoneurons in mixed cultures to a saturating concentration of kainate, $\mathrm{a} \mathrm{G}_{2}$ agonist, was more than $4 \times$ that of motoneurons in sorted cultures. The response of motoneurons in mixed cultures to the $G_{1}$ agonist aspartate was about $200 \mathrm{pA}$, whereas, as demonstrated previously (O'Brien and Fischbach, 1986b), no aspartate current could be evoked in sorted motoneurons.

Table 1. Peak inward current (pA) evoked in motoneurons by $G_{1}$ and $G_{2}$ receptor agonists

\begin{tabular}{lll} 
Agonist & Sorted & Unsorted \\
\hline Aspartatc $\left(10^{-3} \mathrm{M}\right)$ & 0 & $196 \pm 42$ \\
& $(n=23)$ & $(n=14)$ \\
Kainate $\left(10^{-3} \mathrm{M}\right)$ & $245 \pm 43$ & $1190 \pm 210$ \\
& $(n=8)$ & $(n=12)$
\end{tabular}

Motoneurons identified after $6 \mathrm{~d}$ in vitro were voltage clamped at $-50 \mathrm{mV}$. The agonists were applied by pressure ejection $(0.5 \mathrm{sec}$ pulse). Values are means \pm SD. 
Table 2. Peak currents (pA) evoked in motoneurons by inhibitory amino acids

\begin{tabular}{|c|c|c|c|c|}
\hline \multirow[b]{2}{*}{ Day } & \multicolumn{2}{|l|}{ Unsorted } & \multicolumn{2}{|l|}{ Sorted } \\
\hline & $\operatorname{GABA}\left(10^{-4} \mathrm{M}\right)$ & Gly $\left(10^{-3} \mathrm{M}\right)$ & $\operatorname{GABA}\left(10^{-4} \mathrm{M}\right)$ & Gly $\left(10^{-3} \mathrm{M}\right)$ \\
\hline 4 & $\begin{array}{l}1164 \pm 280 \\
(n=11)\end{array}$ & - & $\begin{array}{l}1209 \pm 230 \\
(n=18)\end{array}$ & - \\
\hline 6 & $\begin{array}{l}1300 \pm 310 \\
(n=14)\end{array}$ & $\begin{array}{l}759 \pm 185 \\
(n=12)\end{array}$ & $\begin{array}{l}1110 \pm 205 \\
(n=21)\end{array}$ & $\begin{array}{l}787 \pm 129 \\
(n=11)\end{array}$ \\
\hline 14 & $\begin{array}{l}1400 \pm 254^{a} \\
(n=24)\end{array}$ & - & - & - \\
\hline
\end{tabular}

Motoneurons were identified at the indicated times and voltage clamped at -50 $\mathrm{mV}$. GABA and glycine were applied by pressure ejection. Cells assayed in $15 \mathrm{~d}$ cultures were not identified as motoneurons. The intracellular electrode solution contained $150 \mathrm{~mm} \mathrm{KCl}$. Values are means $\pm \mathrm{SD}$.

${ }^{a}$ These cells were not identified motoneurons.

To be certain that we had not selected a subpopulation of motoneurons during the process of cell sorting that were insensitive to glutamate, unlabeled spinal cord cells were added to cultures of sorted motoneurons $24 \mathrm{hr}$ after the latter were plated. Sorted motoneurons located $4 \mathrm{~d}$ later by fluorescence microscopy were as sensitive to $10^{-3} \mathrm{M}$ glutamate $(300 \pm 40 \mathrm{pA} ; n=$ 8 ), as were unsorted motoneurons in mixed cultures assayed at the same time $(328 \pm 51 \mathrm{pA} ; n=9)$.

When labeled motoneurons are passed through the FACS IV, they are separated from glia and other supporting cells present in the spinal cord as well as from interneurons. Two experiments indicate that the increased sensitivity of motoneurons in mixed cultures is due to interneurons rather than to non-neuronal cells. First, sorted motoneurons were added to cultures that contained proliferating cells dissociated from E15 spinal cords and isolated from neurons by repeated passage in vitro. Second, unsorted spinal cord cultures were exposed to $10^{-5} \mathrm{M}$ ara $\mathrm{C}$ between 24 and $60 \mathrm{hr}$ after plating to eliminate dividing cells. Ara C-treated cultures contained no flat polygonal cells that ordinarily form a confluent monolayer by this time (see Fischbach and Nelson, 1977, Fig. 3). The marked disparity in glutamate currents was evident whether sorted motoneurons were added to non-neuronal cells (Fig. $2 A$ ) or non-neuronal cells were eliminated from mixed spinal cord cell cultures (Fig. $2 B$ ). Hence, we conclude that interneurons are responsible for the induction of chemosensitivity.

Medium conditioned by cultured spinal cord cells did not increase the glutamate sensitivity of sorted motoneurons. Fullstrength conditioned medium or conditioned medium diluted $1: 1$ with fresh medium was added to sorted motoneuron cultures

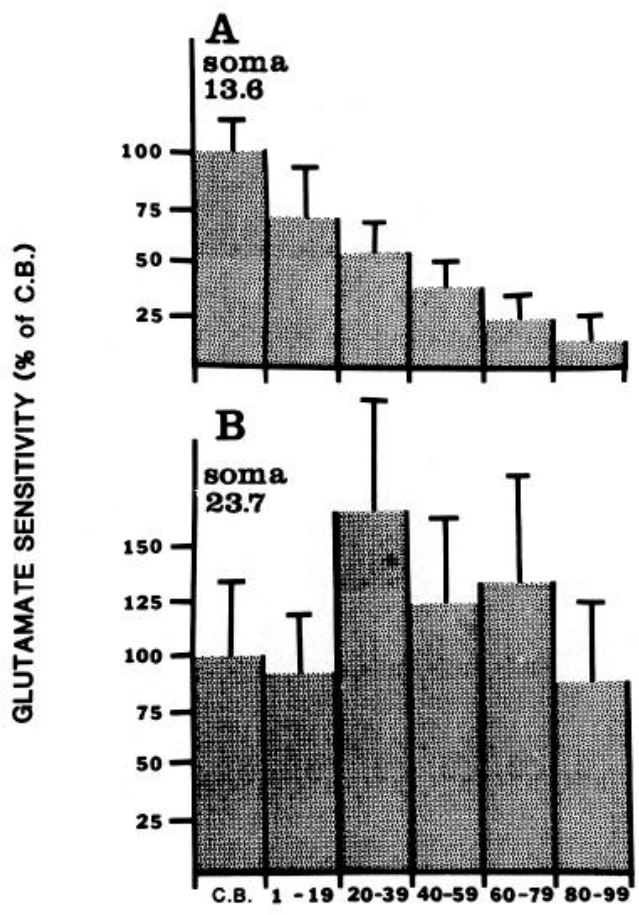

DISTANCE FROM SOMA (microns)

Figure 3. Distribution of glutamate sensitivity determined by iontophoresis. Motoneurons were examined $6 \mathrm{~d}$ after plating. The histograms represent results pooled from 5 sorted motoneurons $(A)$ and 10 motoneurons in mixed spinal cord cultures $(B)$. Each bar represents the mean ratio of the sensitivity recorded within the indicated interval to the mean sensitivity recorded at the soma (mean $\pm \mathrm{SD}$ ).

every $24 \mathrm{hr}$ between day 1 and day 4 in culture. In 3 separate platings the peak inward current evoked by $10^{-4} \mathrm{M}$ glutamate in sorted motoneurons treated with conditioned medium was $46 \pm 6 \mathrm{pA}$ (mean $\pm \mathrm{SD} ; n=27)$, while the peak current in sorted motoneurons treated with unconditioned medium was $40 \pm 3 \mathrm{pA}(n=18)$.

The distribution of glutamate sensitivity along proximal processes of motoneurons was mapped with iontophoretic current pulses of 3-5 msec duration and 100-200 nA amplitude. Movement of the electrode $10 \mu \mathrm{m}$ away from a neurite in the horizontal plane led to a $50 \%$ reduction in the amplitude of the inward current (recorded at the soma); a displacement of $15 \mu \mathrm{m}$

Table 3. Effect of $\gamma$ GG on motoneuron chemosensitivity (pA)

\begin{tabular}{|c|c|c|c|c|}
\hline \multirow[b]{2}{*}{ Agonist } & \multicolumn{2}{|l|}{ Unsorted } & \multicolumn{2}{|l|}{ Sorted } \\
\hline & Control & Treated & Control & Treated \\
\hline Glutamate $\left(10^{-3} \mathrm{M}\right)$ & $\begin{array}{l}210 \pm 27 \\
(n=24)\end{array}$ & $\begin{array}{l}425 \pm 90 \\
(n=25)\end{array}$ & $\begin{array}{l}75 \pm 15 \\
(n=20)\end{array}$ & $\begin{array}{l}150 \pm 350 \\
(n=18)\end{array}$ \\
\hline Kainate $\left(10^{-3} \mathrm{M}\right)$ & $\begin{array}{l}620 \pm 190 \\
(n=19)\end{array}$ & $\begin{array}{l}1300 \pm 295 \\
(n=10)\end{array}$ & $\begin{array}{l}245 \pm 45 \\
(n=8)\end{array}$ & $\begin{array}{l}510 \pm 68 \\
(n=8)\end{array}$ \\
\hline Aspartate $\left(10^{-3} \mathrm{M}\right)$ & $\begin{array}{l}90 \pm 20 \\
(n=9)\end{array}$ & $\begin{array}{l}160 \pm 35 \\
(n=14)\end{array}$ & $\begin{array}{c}0 \\
(n=5)\end{array}$ & $\begin{array}{c}30 \pm 11 \\
(n=13)\end{array}$ \\
\hline $\operatorname{GABA}\left(10^{-4} \mathrm{M}\right)$ & $\begin{array}{l}920 \pm 170 \\
(n=8)\end{array}$ & $\begin{array}{l}862 \pm 140 \\
(n=11)\end{array}$ & $\begin{array}{l}1000 \pm 190 \\
(n=12)\end{array}$ & $\begin{array}{l}970 \pm 210 \\
(n=10)\end{array}$ \\
\hline
\end{tabular}

Sorted and unsorted motoneurons were grown with $10 \mathrm{~mm} \gamma \mathrm{GG}$ for $3 \mathrm{~d}$, as described in Materials and Methods. Their responses to the indicated agonists were assayed on day 4 . In these experiments, unsorted cultures were treated with ara $\mathrm{C}$ to eliminate glia. Values are means $\pm \mathrm{SD}$. 


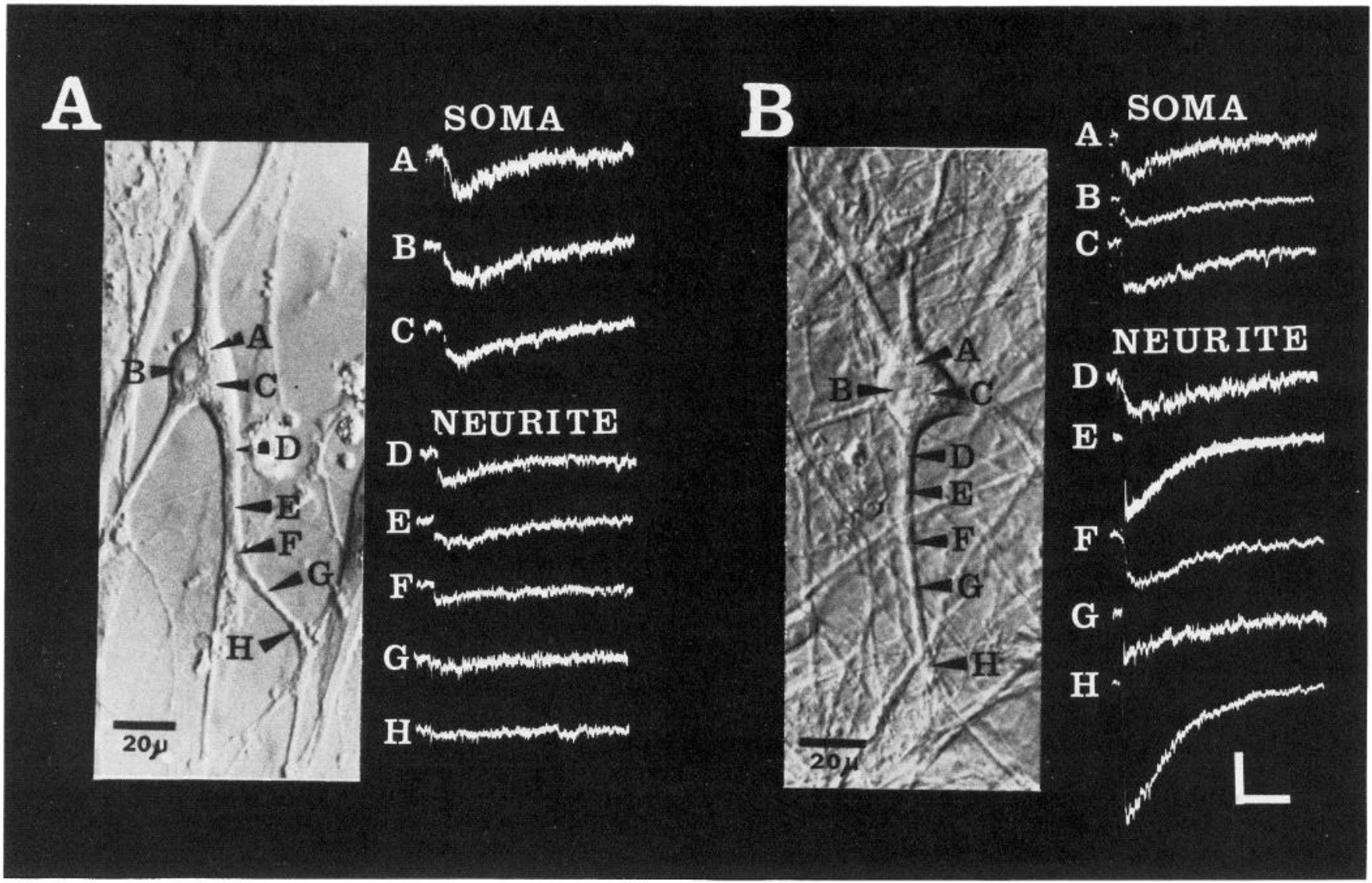

Figure 4. Examples of glutamate currents evoked at several sites on the soma and major processes of a sorted motoneuron $(A)$ and an unsorted motoneuron grown with other spinal cord cells $(B)$. The sensitivity declines steadily with distance from the sorted motoneuron soma, but it remains high along the process of the unsorted motoneuron. Note the 2 hot spots $(E$ and $H)$ along the latter process. Both cells were examined $6 \mathrm{~d}$ after plating. Calibration bars: $20 \mathrm{pA}, 100 \mathrm{msec}$.

led to a $75 \%$ reduction. Figure 3 summarizes results from 9 unsorted and 5 sorted motoneurons. Dendrites of sorted motoneurons were less sensitive than the cell bodies, but the dendrites of motoneurons cultured with other spinal cord cells were usually more sensitive than the somas. Although not obvious in the histograms of Figure 3, the sensitivity along dendrites of unsorted motoneurons was not uniform: Sharp peaks were separated by regions of lower sensitivity (Fig. $4 B$ ). In contrast, no "hot spots" were detected along dendrites of sorted motoneurons: Responses to a fixed glutamate pulse decreased steadily with distance from the soma (Fig. $4 A$ ).

Table 4. Effect of chronic treatment with GPT on amino acid sensitivity of motoneurons

\begin{tabular}{clllll} 
& Unsorted & & & Sorted & \\
\cline { 2 - 3 } \cline { 5 - 6 } Agonist & Control & Treated & & Control & Treated \\
\hline Glutamate & $240 \pm 40$ & $350 \pm 72$ & & $82 \pm 20$ & $141 \pm 39$ \\
$\left(10^{-3} \mathrm{M}\right)$ & $(n=8)$ & $(n=11)$ & & $(n=10)$ & $(n=14)$ \\
Aspartate & $70 \pm 20$ & $150 \pm 55$ & & 0 & $31 \pm 16$ \\
$\quad\left(10^{-3} \mathrm{M}\right)$ & $(n=7)$ & $(n=11)$ & & $(n=4)$ & $(n=14)$ \\
Kainate & $690 \pm 105$ & $1010 \pm 210$ & $268 \pm 62$ & $390 \pm 80$ \\
$\left(10^{-3} \mathrm{M}\right)$ & $(n=14)$ & $(n=12)$ & & $(n=7)$ & $(n=8)$
\end{tabular}

Cultures of sorted and unsorted motoneurons were treated with GPT (10 units/ $\mathrm{ml}$ ) plus $10 \mathrm{~mm}$ pyruvate for $3 \mathrm{~d}$ as described in Materials and Methods. Glutamine was omitted from the medium. The agonists were applied on day 4 in vitro at the indicated concentrations. Values are means \pm SD.
Focal iontophoresis confirmed our conclusion based on pressure ejection of glutamate that the cell bodies of sorted motoneurons were less sensitive than the cell bodies of unsorted motoneurons (Fig. 3). This disparity was evident despite the fact that the 5 sorted neurons selected for study by iontophoresis were among the top $25 \%$ of this group in terms of overall glutamate sensitivity.

We also assayed currents generated by inhibitory amino acids.
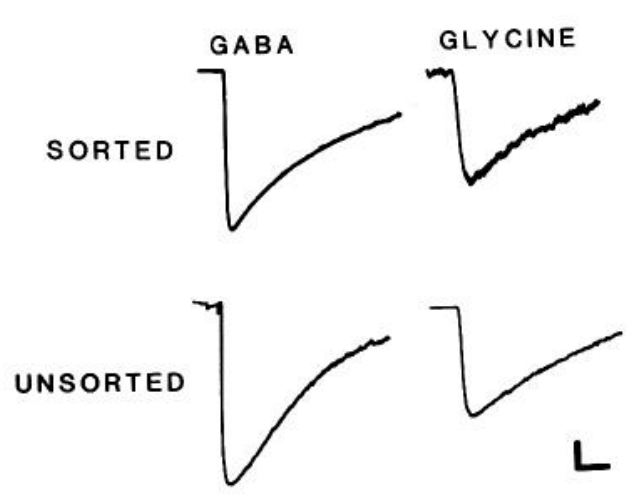

Figure 5. Currents evoked by inhibitory amino acids in sorted and unsorted motoneurons are similar in amplitude. The neurons, assayed $6 \mathrm{~d}$ after plating, were clamped at $-50 \mathrm{mV}$. The intracellular electrode contained $150 \mathrm{~mm} \mathrm{KCl}$. GABA was applied by pressure ejection at $10^{-4}$ $\mathrm{M}$ and glycine at $10^{-3} \mathrm{M}$. Each trace was obtained from a different cell. Calibration bars: 100 pA, 2 sec. 


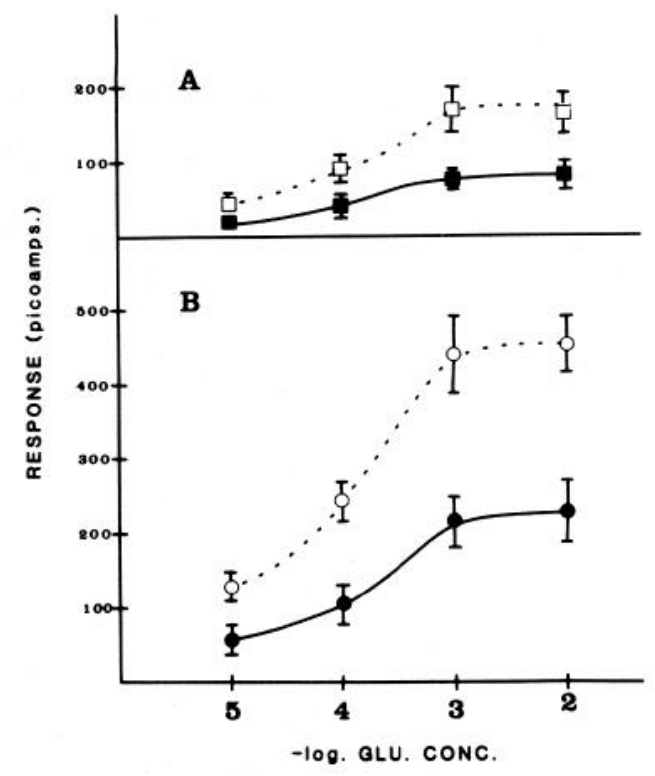

Figure 6. Chronic exposure to $\gamma \mathrm{GG}$ increases motoneuron glutamate sensitivity. Sorted $(A)$ and unsorted $(B)$ motoneurons were cultured in the presence (open symbols) and absence (closed symbols) of $10 \mathrm{~mm}$ $\gamma \mathrm{GG}$ for $3 \mathrm{~d}$ and assayed on day 4 . Each point represents the mean \pm $\mathrm{SD}$ of at least 10 cells, all of which were voltage clamped at $-60 \mathrm{mV}$.

As shown in Figure 5, GABA and glycine induced inward currents $(150 \mathrm{~mm} \mathrm{KCl}$ replaced $\mathrm{K}$-acetate in the recording electrode) of equal magnitude in sorted and unsorted motoneurons in $6 \mathrm{~d}$ cultures. Results from all cells tested are summarized in Table 2. Inhibitory synapses form (or become functional) later than excitatory synapses in our culture system. All motoneurons tested in $6 \mathrm{~d}$ cultures exhibited EPSCs, but spontaneous IPSCs were detected in less than $10 \%$ of the motoneurons at this time (O'Brien and Fischbach, 1986c). Thus, it is possible that GABA responses increase in unsorted motoneurons after the first week in culture. Because the fluorescent label was lost with time, we could not identify motoneurons reliably in heterogeneous spinal cord cell cultures after 1 week in vitro. However, the GABA sensitivity of the largest neurons in $15 \mathrm{~d}$ cultures was not significantly different from that of sorted motoneurons in $6 \mathrm{~d}$ cultures (Table 2). All of the neurons tested in 14-15 d cultures exhibited IPSCs.

\section{Regulation of motoneuron glutamate sensitivity by agonists}

Interneurons might regulate the glutamate chemosensitivity of chick motoneurons through the release of glutamate itself. If this were the case, an inhibitor of $G_{1}$ and $G_{2}$ receptors such as $\gamma \mathrm{GG}$ would be expected to prevent the observed increase in

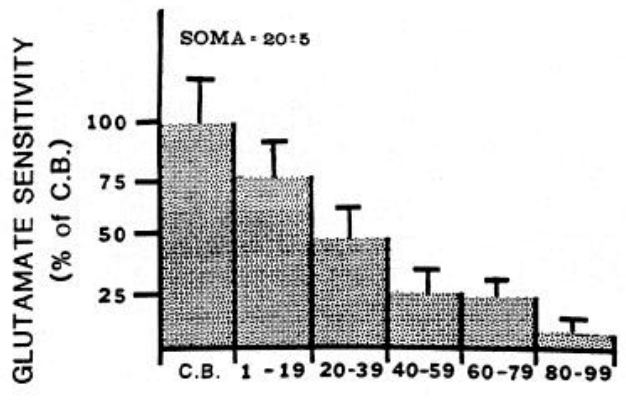

DISTANCE FROM SOMA (microns)

Figure 7. $\gamma \mathrm{GG}$ does not alter the distribution of chemosensitivity in sorted motoneurons. The histogram includes recordings from 5 sorted motoneurons grown in the presence of $10 \mathrm{nM} \gamma \mathrm{GG}$ for $3 \mathrm{~d}$ (compare with Fig. 3). Each bar indicates the mean peak inward current within the indicated interval relative to the mean soma inward current (mean \pm SD).

sensitivity. However, when motoneurons were grown in medium supplemented with $10 \mathrm{~mm} \gamma \mathrm{GG}$ from day 2 to 4 after plating, the inductive effect of interneurons on motoneuron glutamate chemosensitivity was still evident (Fig. 6). The marked induction was superimposed on significant increases in the sensitivity of both unsorted and sorted motoneurons compared with cells grown in control medium (Table 3 ). Thus, glutamate cannot be mediator of the interneuron effect. On the contrary, this amino acid apparently down-regulates its own receptors. The down-regulation involved both $G_{1}$ and $G_{2}$ receptors, as responses to specific $G_{1}$ and $G_{2}$ agonists were increased following incubation in $\gamma \mathrm{GG}$ (Table 3). The effect of $\gamma \mathrm{GG}$ was specific in that no effect on the sensitivity of motoneurons to saturating concentrations of GABA was evident (Table 3).

Despite the increase in overall glutamate sensitivity, $\gamma \mathrm{GG}$ did not alter the distribution of sensitivity along the major processes of sorted motoneurons. Figure 7 illustrates the response profile of 5 sorted motoneurons grown with $\gamma \mathrm{GG}$. The steady decline with distance from the soma is comparable to that of sorted motoneurons in control medium (see Fig. 3).

The fact that glutamate responses of sorted motoneurons increased following long-term exposure to $\gamma \mathrm{GG}$ makes it unlikely that the down-regulation of receptors is due solely to synaptically released glutamate. This does not, however, rule out glutamate as the agent responsible for down-regulation because glutamate is present in the culture medium. Interneurons and glia accumulate glutamate, so their absence from cultures of sorted motoneurons might result in a relatively high extracellular concentration of this amino acid. Indeed, we found that the concentration of glutamate in medium removed from cultures

Table 5. Effect of chronic treatment with 2-APV or kainate on motoneuron $G_{1}$ and $G_{2}$ currents (pA)

\begin{tabular}{|c|c|c|c|c|c|c|}
\hline \multirow[b]{3}{*}{ Agonist } & \multicolumn{4}{|l|}{ Unsorted } & \multirow{2}{*}{\multicolumn{2}{|c|}{ Sorted + APV }} \\
\hline & \multicolumn{2}{|l|}{$\overline{\mathrm{APV}}$} & \multicolumn{2}{|l|}{ Kainate } & & \\
\hline & Control & Treated & Control & Treated & Control & Treated \\
\hline Aspartate $\left(10^{-3} \mathrm{M}\right)$ & $\begin{array}{c}85 \pm 23 \\
(n=13)\end{array}$ & $\begin{array}{l}240 \pm 65 \\
(n=28)\end{array}$ & $\begin{array}{c}78 \pm 15 \\
(n=10)\end{array}$ & $\begin{array}{l}65 \pm 19 \\
(n=8)\end{array}$ & 0 & $\begin{array}{c}46 \pm 14 \\
(n=30)\end{array}$ \\
\hline Kainate $\left(10^{-3} \mathrm{M}\right)$ & $\begin{array}{l}710 \pm 89 \\
(n=13)\end{array}$ & $\begin{array}{l}780 \pm 140 \\
(n=28)\end{array}$ & $\begin{array}{l}730 \pm 183 \\
(n=12)\end{array}$ & $\begin{array}{l}220 \pm 70 \\
(n=21)\end{array}$ & $\begin{array}{l}240 \pm 58 \\
(n=12)\end{array}$ & $\begin{array}{l}240 \pm 27 \\
(n=9)\end{array}$ \\
\hline
\end{tabular}

Motoneurons grown in mixed spinal cord cell cultures were treated for $3 \mathrm{~d}$ with $1 \mathrm{~mm} 2$-APV or for $2 \mathrm{~d}$ with $50 \mu \mathrm{M}$ kainate. Cultures of sorted motoneurons were treated with 2-APV only. Peak currents were determined on day 4 in vitro. Values are means $\pm \mathrm{SD}$. 
of sorted motoneurons $(61 \pm 19 \mu \mathrm{M} ; n=4)$ was 5 times greater than the concentration in medium from unsorted cultures $(14 \pm$ $2 \mu \mathrm{M} ; n=4)$.

Further evidence that glutamate is responsible for receptor down-regulation was obtained by adding glutamate-pyruvate transminase (GPT) to sorted and unsorted cultures. This enzyme, which normally converts alanine and $\alpha$-ketoglutarate to pyruvate and glutamate, respectively, can, in the presence of a high concentration of pyruvate, convert glutamate to $\alpha$-ketoglutarate. In these experiments, L-glutamine was omitted from the medium to decrease glutamate synthesis. When sorted or unsorted cultures were incubated with GPT and $10 \mathrm{~mm}$ pyruvate, the levels of glutamate were drastically reduced $(0.5 \pm 0.2$ $\mu \mathrm{M}, n=4 ; 0.6 \pm 0.3 \mu \mathrm{M}, n=4$, respectively), and there was an increase in glutamate sensitivity in each case. Both $G_{1}$ and $G_{2}$ receptors were affected (Table 4). Addition of $10 \mathrm{~mm}$ pyruvate without the enzyme had no effect on glutamate currents. It should be noted that despite the equivalently low levels of excitatory amino acids in sorted and unsorted cultures treated with GPT, the inductive effect of interneurons on $G_{1}$ and $G_{2}$ currents was still evident (Table 4 ).

$G_{1}$ and $G_{2}$ receptors could be regulated independently. Table 5 presents data from motoneurons in mixed cultures incubated in the presence of the specific $G_{1}$ receptor antagonist 2-APV for $3 \mathrm{~d}$. These cells showed a 3 -fold increase in $\mathrm{G}_{1}$ currents induced by aspartate, without a detectable change in $\mathrm{G}_{2}$ currents evoked by kainic acid. In cultures of sorted motoneurons, 2-APV brought out a small but definite increase in aspartate sensitivity, with no change in kainate sensitivity. Independent regulation of $\mathrm{G}_{2}$ receptors was demonstrated with the specific $G_{2}$ agonist kainate by incubating spinal cord cultures containing labeled motoneurons with $50 \mu \mathrm{M}$ kainate for $2 \mathrm{~d}$. After the kainate was washed out, $G_{2}$ currents were reduced 3 -fold but $G_{1}$ currents were unchanged (Table 5).

\section{Discussion}

Glutamate, applied by pressure ejection, generates much larger currents in motoneurons cultured with other spinal cord cells than in sorted motoneurons grown without them. The increase in chemosensitivity along the major processes of unsorted motoneurons is particularly dramatic. It is unlikely that the increase in sensitivity is due to an increase in the affinity of receptors for glutamate, because the $\mathrm{ED}_{50}$ 's of sorted and unsorted motoneurons are identical. It is also unlikely that the increase reflects a change in single-channel conductance, mean channel open time, or ion selectivity, because the power spectra of glutamate current fluctuations and the glutamate reversal potentials of sorted and unsorted motoneurons are the same in sorted and unsorted motoneurons (O'Brien and Fischbach, 1986b). [The glutamate response of sorted motoneurons is due predominantly to activation of $G_{2}$ receptors (O'Brien and Fischbach, 1986b). The $\mathrm{G}_{2}$ component of the spectrum of unsorted motoneuron glutamate current fluctuations is identical to the spectrum recorded in sorted cells.] Thus, the increase in sensitivity must be due to an increase in the number of open channels. This, in turn, might be due to an increase in the number of receptors (binding sites) or in the probability of channel opening. Recognizing this caveat, we shall refer to the number of (open) channels, rather than the number of receptors.

The dendritic arbor of motoneurons grown with other spinal cord cells is more extensive than that of motoneurons grown in isolation (O'Brien and Fischbach, 1986a). Thus, a greater area of membrane must be exposed to glutamate applied by pressure ejection. However, this factor cannot account for the observed increase in chemosensitivity. First, the increase in total neuritic length was less than 2-fold, while the increase in inward current following the standard pressure pulse was greater than 6 -fold. Second, the glutamate response of sorted motoneurons is ap- parently due entirely to $G_{2}$ receptors, whereas motoneurons grown in the presence of interneurons express both $G_{1}$ and $G_{2}$ receptors. Third, no change in GABA currents was detected. An increased GABA response would be expected if the change in glutamate sensitivity was simply due to a greater membrane area. Finally, iontophoresis of glutamate indicates that the local receptor density over the soma and major processes of unsorted motoneurons is increased compared with that of sorted motoneurons.

The increase in channel number is probably due to the presence of interneurons rather than to glia. This depends on the assumptions that all proliferating cells are eliminated by ara $\mathrm{C}$ and that all ara $\mathrm{C}$-resistant cells are neurons. The light-microscopic appearance and electrophysiological properties of cells in ara C-treated cultures suggest that these assumptions are justified. The mechanism by which interneurons increase the number of glutamate channels remains to be determined. They may release 1 or more factors that increase receptor number or receptor-ionophore coupling. We did not detect an effect of spinal cord conditioned medium on glutamate sensitivity, but this negative result does not rule out the existence of soluble inducers. Their concentration in conditioned medium may simply be too low, or they may be masked by inhibitory factors. Cell cultures of sorted motoneurons should prove useful in attempts to isolate and characterize the factors involved. It will be important to determine if they are similar to those that increase the number and aggregation of $\mathrm{ACh}$ receptors in skeletal myotubes (Buc-Caron et al., 1983; Jessell et al., 1979; Usdin and Fischbach, 1984, 1986).

The inductive effect of interneurons cannot be mediated by glutamate. On the contrary, experiments with $\gamma \mathrm{GG}$, GPT, and 2-APV indicate that the glutamate sensitivity of motoneurons is inversely related to the effective concentration of glutamate in the medium. It is unlikely that the action of intcrncuroninducing factors is simply the reciprocal of that by which agonists down-regulate glutamate sensitivity. Motoneurons grown with interneurons were 3-5 times more sensitive than sorted motoneurons whether the effective concentration of glutamate in the medium was low (reduced by GPT or blocked by $\gamma$ GG) or high (following exposure to ara $\mathrm{C}$ ). In addition, there is an important distinction between the increase in chemosensitivity mediated by interneurons and down-regulation by agonists. Interneurons increase the sensitivity along major processes, whereas reduction of glutamate-receptor interaction with $\gamma \mathrm{GG}$ does not.

Spontaneous EPSCs were detected in nearly all of the motoneurons tested in 5-6 d cultures, so it is reasonable to suppose that receptor-inducing factors are released at excitatory synaptic boutons. The striking increase in sensitivity along major processes is interesting in this regard because excitatory inputs to motoneurons in several species are distributed throughout the dendritic arbor (Fadiga and Brookhart, 1960; Jack et al., 1971; Rall et al., 1967; Redman and Walmsley, 1983). Determination of the precise domain of influence of individual synaptic boutons may help settle this issue. Unfortunately, nothing is known of the distribution of excitatory amino acid receptors on the scale of individual boutons on motoneurons or any other neuron within the CNS. We found relative peaks of sensitivity along motoneuron processes, but the resolution of our mapping technique was only $10-15 \mu \mathrm{m}$. Smaller and shorter pulses must be used to delineate the size of hot spots and their relation to synaptic boutons. Ransom et al. (1977) found hot spots along processes of mouse spinal cord cells with iontophoretic pulses comparable to those used here. When the iontophoretic pipette was positioned at a hot spot, the glutamate pulse often triggered a shower of EPSPs, suggesting that boutons were in the immediate vicinity.

Modulation of postsynaptic receptors may determine the efficacy of synapses formed on embryonic motoneurons. As 
shown in the preceding paper (O'Brien and Fischbach, 1986c), relatively few glutamate channels are activated at interneuronmotoneuron synapses during the first week in vitro. Therefore, a small increase in the number of open channels at individual boutons might have a large effect on synaptic transmission at this stage of development. Modulation of postsynaptic receptor density may regulate synaptic efficacy in adult motoneurons as well. Synapses located on relatively distal dendritic branches depolarize the soma as effectively as do more proximal contacts (Iansek and Redman, 1973; Jack et al., 1971). One explanation for this apparent paradox is that distal synapses generate larger synaptic currents. If the amount of transmitter released saturates the postsynaptic receptors, the increased current might reflect a relatively high receptor density, and this, in turn, might be regulated by the presynaptic terminals.

\section{References}

Buc-Caron, M.-H., P. Nystrom, and G. D. Fischbach (1983) Induction of acetylcholine receptor synthesis and aggregation: Partial purification of low molecular weight activity. Dev. Biol. 95: 378-386.

Cohen, M. W. (1980) Development of an amphibian neuromuscular junction in vivo and in culture. J. Exp. Biol. 89: 43-56.

Fadiga, E., and J. M. Brookhart (1960) Monosynaptic activation of different portions of the motoneuron membrane. Am. J. Physiol. 198: 693-703.

Fambrough, D. M. (1979) Control of acetylcholine receptors in skeletal muscle. Physiol. Rev. 59: 165-227.

Fischbach, G. D., and P. G. Nelson (1977) Cell culture in neurobiology. In Handbook of Physiology: The Nervous System, Vol. 1, E. Kandel, ed., pp. 719-774, American Physiological Society, Bethesda, MD.

Fischbach, G. D., E. Frank, T. M. Jessell, L. L. Rubin, and S. M. Schuetze (1979) Accumulation of acetylcholine receptors and acetylcholinesterase at newly formed nerve-muscle synapses. Pharmacol. Rev. 30: 411-428.

Iansek, R., and S. J. Redman (1973) The amplitude, time course and charge of unitary excitatory post-synaptic potentials evoked in spinal motoneuron dendrites. J. Physiol. (Lond.) 234: 665-688.
Jack, J. J. R., S. Miller, R. Porter, and S. J. Redman (1971) The time course of minimal excitatory post-synaptic potentials evoked in spinal motoneurons by group Ia afferents. J. Physiol. (Lond.) 215: 353-380.

Jessell, T. M., R. E. Siegel, and G. D. Fischbach (1979) Induction of acetylcholine receptors on cultured skeletal muscle by a factor extracted from brain and spinal cord. Proc. Natl. Acad. Sci. USA 76: 5397-5401.

Jones, B. N., and J. P. Gilligan (1983) O-Phthaldialdehyde precolumn derivatization and reversed phase high performance liquid chromatography of polypeptide hydrolysates and physiological fluids. J. Chromatogr. 266: 471-482.

O'Brien, R. J., and G. D. Fischbach (1986a) Isolation of embryonic chick motoneurons and their survival in vitro. J. Neurosci. 6: $3265-$ 3274.

O'Brien, R. J., and G. D. Fischbach (1986b) Characterization of excitatory amino acid receptors expressed by embryonic chick motoneurons in vitro. J. Neurosci. 6: 3275-3283.

O'Brien, R. J., and G. D. Fischbach (1986c) Excitatory synaptic transmission between interneurons and motoneurons in chick spinal cord cell cultures. J. Neurosci. 6: 3284-3289.

Rall, W., R. E. Burke, T. G. Smith, P. G. Nelson, and K. Frank (1967) Dendritic location of synapses and possible mechanisms for the monosynaptic EPSP in motoneurons. J. Neurophysiol. 30: 11691193.

Ransom, B. R., P. N. Bullock, and P. G. Nelson (1977) Mouse spinal cord in cell culture. III. Neuronal chemosensitivity and its relationship to synaptic activity. J. Neurophysiol. 40: 1163-1177.

Redman, S. J., and B. Walmsley (1983) The time course of synaptic potentials evoked in cat spinal motoneurons at identified group la synapses. J. Physiol. (Lond.) 343: 117-133.

Steinbach, J. H., and R. J. Bloch (1986) The distribution of acetylcholine receptors on vertebrate skeletal muscle cells. In Receptors in Biological Systems, R. M. Gorezynski and G. B. Price, eds., Academic, New York.

Usdin, T. B., and G. D. Fischbach (1984) Acetylcholine receptor synthesis inducing factor. Soc. Neurosci. Abstr. 10:925.

Usdin, T. B., and G. D. Fischbach (1986) Purification and characterization of a polypeptide from chick brain that promotes the accumulation of acetylcholine receptors in chick myotubes. J. Cell Biol. 103: 493-508. 